JOURNAL OF

SYMPLECTIC GEOMETRY

Volume 2, Number 1, 83 - 107, 2003

\title{
A NEW CONSTRUCTION OF POISSON MANIFOLDS
}

\author{
A. Ibort and D. Martínez Torres
}

A new technique is presented for construction of Poisson manifolds. This technique is inspired by surgery ideas used to define Poisson structures on 3-manifolds and Gompf's surgery construction for symplectic manifolds. As an application of these ideas it is proved that for all $n \geq d \geq 4, d$ even, any finitely presentable group is the fundamental group of a $n$-dimensional orientable closed Poisson manifold of constant rank $d$. The unimodularity of some of the Poisson structures thus constructed is studied.

Keywords: Poisson manifolds, Poisson surgery, modular class, fundamental groups

MSC 2000 Classification: 57R17, 53D17, 57R65

\section{Introduction}

The use of almost-complex methods in symplectic geometry together with new surgery techniques has notably increased our understanding of the topology of symplectic manifolds (see for instance the foundational papers $[\mathbf{5}, \mathbf{2}, \mathbf{4}])$. These results constitute an extraordinary mixture of "soft" and "hard" mathematical ideas in the sense of Gromov [6]. In spite of all this success very little is known about nontrivial families of symplectic manifolds. Families of symplectic manifolds lead naturally to the notion of Poisson manifolds.

A Poisson structure on a manifold $M$ is a Poisson algebra structure on its sheaf of functions. That is to say, given two local functions $f, g$ on $M$ we define on their common domain of definition a bilinear bracket $\{f, g\}$ satisfying the following properties: 
1) skew symmetry, $\{f, g\}=-\{g, f\}$.

2) Leibniz rule, $\{f, g h\}=g\{f, h\}+\{f, g\} h$.

3) Jacobi identity, $\{f,\{g, h\}\}+\{g,\{h, f\}\}+\{h,\{f, g\}\}=0$.

Alternatively, a Poisson structure on a manifold $M$ is given by a bivector $\Lambda$ such that $[\Lambda, \Lambda]=0$, where $[\cdot, \cdot]$ denotes the Schouten bracket (see for instance [10] and references therein). The Poisson bracket $\{f, g\}$ of two functions is given in terms of $\Lambda$ by $\Lambda(d f, d g)$. Moreover, the Poisson tensor $\Lambda$ defines a natural bundle morphism $\#: T^{*} M \rightarrow T M$ whose range defines an involutive distribution $\mathcal{S}_{\Lambda}$ whose integral leaves are equipped with a canonical symplectic structure. Conversely, any foliation $\mathcal{S}$ by symplectic manifolds of a manifold $M$ such that for any smooth function the hamiltonians of the restriction of the function to each leaf glue into a smooth vector field, induces a unique Poisson structure whose symplectic foliation is precisely $\mathcal{S}$ [10]. This makes precise the idea mentioned above, that Poisson structures on manifolds provide the geometrical setting for the description of smooth families of symplectic structures.

Looking at the known examples of Poisson manifolds, we see that in most occassions we start with an algebraic structure (a Lie algebra, a cocycle, etc.) and then we construct a manifold whose Poisson structure is related to the initial algebraic one. However it would be most interesting to explore the converse viewpoint. Given a manifold $M$, determine the nontrivial Poisson structures that it supports. This is of course an extremely difficult task because of the intrinsic non-linearity of Poisson structures. Some work in this direction has been already done by M. Bertelson who has studied in [1] the problem of characterizing regular foliations which arise from Poisson structures. In order to accomplish part of this task, another step worth taking is to check the possibility of extending smooth topological constructions to the Poisson category. Some of them have already been carried over to the symplectic setting: D. McDuff [8] has defined the blowing up of a symplectic submanifold and R. Gompf [4] has used the normal connected sum to construct symplectic manifolds with arbitrary fundamental group. In this sense symplectic geometry is "flexible", in sharp contrast to Kähler geometry. Of course, trivial families of symplectic manifolds can be constructed just by taking the product of a symplectic manifold $M$ with an arbitrary compact manifold $Q$. Such trivial product Poisson structures will have the same fundamental group as $M$ provided 
that $\pi_{1}(Q)=0$. Hence unless we are looking for one-parameter families of symplectic manifolds, the existence of families with arbitrary fundamental group is trivial (which is not to say that their classification is also trivial, of course). Thus the problem of constructing Poisson manifolds with arbitrary fundamental group is reduced to the particular situation of codimension one symplectic foliations, and more concretely to the search for 5-dimensional compact Poisson manifolds of constant rank 4 with arbitrary fundamental group. In the process of constructing them, we will introduce a surgery operation for Poisson manifolds that naturally extends Gompf's construction. We will show in addition some properties of some of the Poisson manifolds obtained in this way which are characteristic of the Poisson category, like the behavior of its modular class [12]. We will also provide various examples exhibiting some of these properties.

We will start by recalling in section 2 how one can endow any compact orientable 3-manifold with a rank 2 regular Poisson structure. This construction, which depends on well-known results on foliations of orientable 3-manifolds, will be adapted in sections 3 and 4 to define a surgery technique that extends Gompf's to the Poisson category. Section 5 is devoted to the study of the modular class of some Poisson manifolds constructed by surgery, and in section 6 we will prove that any finitely presentable group can be realized as the fundamental group of a compact regular Poisson manifold of dimension $n$ and even rank $d$ with $n \geq d \geq 4$.

\section{Poisson structures on 3-dimensional manifolds}

A regular Poisson structure on a 3 -manifold $M^{3}$ is just a foliation by surfaces with a leafwise smooth area form. In particular, if $M^{3}$ is orientable, finding such a structure turns out to be an easy problem of differential topology, whose non-trivial part is to endow the manifold with the foliation. The problem of finding a codimension one foliation on an oriented 3-manifold is a classical one which is already solved. We now give an outline of a solution (in which we do not ask much of the foliation), because it essentially contains the ideas that give rise to a surgery construction for Poisson manifolds.

Recall that every orientable compact 3-manifold $M$ can be obtained from $S^{3}$ by surgery on a link whose components $k_{j}$ can be chosen as close to the unknot as we want. Moreover, the framings are of the form

$\left(m_{j} \pm 1 l_{j}\right)$, where $m_{j}, l_{j}$ are the meridian and longitude of the boundary 
tori. Since the components of the link can be chosen to be very close to the unknot, they can be made transversal to the Reeb foliation $\mathcal{R}$ of $S^{3}$, i.e., the knots are submanifolds transversal to the leaves inheriting the trivial Poisson structure. Once open tubular neighbourhoods of $k_{j}$ have been removed from the solid tori $T_{j}=D^{2} \times S^{1}$ to be glued, the boundaries of the leaves of $\mathcal{R}$ will be non-separating curves on $\partial T_{j}$, cutting the meridian once. These curves are non-trivial in the homology of $T_{j}$, so we cannot hope to add a punctured surface to get closed leaves. However, if we remove a small tubular neighbourhood $\mathcal{N}_{j}$ of the longitude $\alpha_{j}=\{0\} \times S^{1}$ we can find a map $\phi_{j}: T_{j}-\mathcal{N}_{j} \rightarrow S^{1} \times I \times S^{1}$ such that the image of the curve $m_{j}$ on $T_{j}$ goes to $S^{1} \times\{0\} \times\{e\}$, the meridian of $S^{1} \times I \times S^{1} \subset D^{2} \times S^{1}$. Hence, pulling back the Reeb foliation of $S^{1} \times I \times S^{1}$ we get a foliation except inside a solid torus, where we again put a Reeb component. We have thus proved the following proposition.

Proposition 1. Every oriented compact 3-manifold admits a regular rank 2 Poisson structure.

Using the ideas above we see that any fibrered knot of a 3-manifold gives a foliation with a Reeb component and a "modified" Reeb component, where instead of having disks approaching the torus we have punctured oriented surfaces (the Seifert surfaces of the knot). However, the previous argument does not allow us to construct for instance a Poisson structure on a 3-manifold realizing as a fundamental group an arbitrary finitely presented group $G$.

\section{Fibrered Poisson structures}

We have seen in the previous section that to perform surgery in an oriented Poisson 3-manifold we do not need to worry about the Poisson tensor itself but only about extending the symplectic foliation we had. It is not difficult to propose a surgery technique for Poisson manifolds which is indeed an extension of the normal connected sum for symplectic manifolds. Roughly speaking, we will use a transversal submanifold that intersects symplectically the leaves of the symplectic foliation. This will allow us to perform the normal connected sum along the symplectic submanifolds and we will show that the resulting manifold admits a Poisson structure determined by the ones we had initially. We will see that with the appropriate setting the proofs will be natural generalizations of those of Gompf [4]. 
3.1. Poisson structures compatible with fibre bundle structures. Let $\pi: P \rightarrow Q$ be a fibre bundle. We call a Poisson structure $\Lambda_{P}$ on $P$ compatible with the fibre bundle structure if the symplectic leaves of $\Lambda_{P}$ are the fibres of $\pi$ (hence the fibres are connected). We will also call the triple $\left(P, \pi, \Lambda_{P}\right)$ a fibrered Poisson manifold. If $P$ is compact this is equivalent to saying that the space of leaves is a smooth manifold $Q$ such that the projection $\pi: P \rightarrow Q$ is a submersion.

We begin by noticing that whenever one has a foliation, one can do the usual exterior calculus in the bundles associated to the distribution. In our case we will have a locally trivial fibration $\pi: P \rightarrow Q$ and the bundle we are interested in is the one of vertical vectors, i.e. the kernel of $\pi$. We will speak of vertical vector fields and $k$-forms, Lie derivatives in the direction of vertical vector fields and the exterior derivative of vertical $k$-forms. We shall denote the set of vertical $k$ forms by $\Omega_{\text {fib }}^{k}(P \rightarrow Q)$, and by $d_{\pi}$ the exterior vertical derivative (or just $d$ if there is no risk of confusion). Recall that one can pull-back vertical forms by fibre bundle morphisms and that any known relation involving Lie derivatives also holds for vertical vector fields and forms (it holds fibrewise and defines a smooth section of the corresponding bundle).

Let us denote the cohomology groups of the complex $\left(\Omega_{\mathrm{fib}}^{*}(P \rightarrow\right.$ $\left.Q), d_{\pi}\right)$ by $H_{\mathrm{fib}}^{k}(P \rightarrow Q)$. We have the corresponding forgetful maps $f: \Omega^{k}(P) \rightarrow \Omega_{\text {fib }}^{k}(P \rightarrow Q)$, and $f: H^{k}(P) \rightarrow H_{\text {fib }}^{k}(P \rightarrow Q)$.

Now it is straightforward to check that a Poisson structure $\Lambda_{P}$ on $P$ compatible with the fibration $\pi: P \rightarrow Q$ is determined by a closed non-singular vertical 2-form $\omega_{P} \in \Omega_{\text {fib }}^{2}\left(P \rightarrow Q\right.$ ) (and hence $\left[\omega_{P}\right] \in$ $H_{\text {fib }}^{2}(P \rightarrow Q)$ ). We will call $\omega_{P}$ the Poisson 2 -form (or just the Poisson form) of $\Lambda_{P}$.

There are some results about the cohomology $H_{\text {fib }}^{*}(P \rightarrow Q)$ that will be used later. We start by recalling that for a closed manifold with a metric, Hodge theory allows one to obtain for any $k$-form $\alpha$ a unique decomposition:

$$
\alpha=d \beta \oplus \delta \eta \oplus \rho
$$

where $\beta$ is coexact, $\eta$ exact and $\rho$ harmonic, and all three are images of $\alpha$ by smooth operators. Moreover we also have relative Hodge theory for a pair $(N, K)$, where $N$ is a compact manifold and $K$ a closed set (that is to say for forms with support contained in $N-K$ ). This 
implies that we also have the above results for a compact manifold $N$ with non-empty boundary and forms with support in the interior on $N$ (to show that this is so it is enough to double the manifold and apply relative Hodge theory).

When $\pi: P \rightarrow Q$ is a locally trivial fibration and $P$ is closed, we can also apply Hodge theory to get the same decomposition above, eq. (1), for vertical $k$-forms. We observe that any metric on $P$ restricts to a metric on each fibre and there we can apply the usual Hodge theory. After gluing what we construct in this way fibrewise, we get again smooth projection operators because in a trivialization we are just working in a fibre with a smooth family of forms and metrics. If $P$ is compact and $\partial P \neq \emptyset$, the relative Hodge theory $(P, \partial P)$ (where we use forms each of whose support does not intersect $\partial P$ ) also holds, because on each trivialization that we use to see that the construction is smooth, the boundaries are setwise identified. As a consequence, for $\pi: P \rightarrow Q$ locally trivial and $P$ closed a vertical closed form on $P$ is exact if and only if it is fibrewise exact. Similarly, if $P$ is compact and $\partial P \neq \emptyset$, a vertical closed form whose support does not intersect $\partial P$, so that the form vanishes on a neighbourhood of the boundary, it is exact if and only if it is exact with potential form vanishing in a neighbourhood of the boundary.

The result also implies that for a smooth family of exact vertical $k$ forms on a compact manifold, one can find a smooth family of vertical $(k-1)$-forms whose exterior derivative is the initial family: to see this consider the vertical forms on the direct sum of the initial bundle and the trivial bundle with rank the number of parameters of the family. If all the $k$-forms of the family vanish in a neighbourhood of the boundary, the $(k-1)$-forms will also vanish in that neighbourhood.

3.2. Transversal Poisson fibrered submanifolds. A smooth Poisson submanifold [11] of a Poisson manifold $\left(M, \Lambda_{M}\right)$ is defined as a triple $\left(P, \Lambda_{P}, j\right)$ where $j:\left(P, \Lambda_{P}\right) \rightarrow\left(M, \Lambda_{M}\right)$ is a Poisson morphism embedding $P$ into $M$. Besides this there are submanifolds $P$ of a Poisson manifold $M$ which inherit a Poisson structure because the foliation of $M$ induces a foliation of $P$ by symplectic submanifolds which fit into a Poisson structure, but where the natural inclusion map is not a Poisson morphism. We will consider submanifolds of Poisson manifolds from this more general perspective. Thus, a Poisson submanifold of a given Poisson manifold $\left(M, \Lambda_{M}\right)$ will be a submanifold intersecting the leaves in symplectic submanifolds and inheriting a Poisson structure 
from $\Lambda_{M}$. The inherited Poisson structure is necessarily unique. These Poisson submanifolds generalize, in a natural way, the symplectic submanifolds of a symplectic manifold. In particular we will be dealing with a special class of Poisson submanifolds compatible with a given fibration.

Definition 1. Let $\left(M, \Lambda_{M}\right)$ be an $n$-dimensional Poisson manifold of rank $d,\left(P, \Lambda_{P}\right)$ a Poisson manifold where $P$ is compact and fibrered over the $(n-d)$-dimensional manifold $Q$, and $\Lambda_{P}$ is compatible with the fibration. An embedding $j: P \rightarrow\left(M, \Lambda_{M}\right)$ is said to embed $\left(P, \Lambda_{P}\right)$ as a transversal Poisson fibrered submanifold of $\left(M, \Lambda_{M}\right)$ if:

i. $j(P)$ is contained in the regular set of $\left(M, \Lambda_{M}\right)$.

ii. $j(P)$ cuts the symplectic leaves of $\left(M, \Lambda_{M}\right)$ transversally.

iii. $j(P)$ inherits a Poisson structure from $\left(M, \Lambda_{M}\right)$ that coincides with $\Lambda_{P}$.

The existence of such a submanifold implies that the symplectic leaves of $\left(M, \Lambda_{M}\right)$ are nicely arranged in a neighbourhood of the submanifold. To be more precise:

Lemma 1. If $j: P \rightarrow\left(M, \Lambda_{M}\right)$ embeds the fibrered Poisson manifold $\left(P, \Lambda_{P}\right) \rightarrow Q$ in $M$ as a codimension $r$ transversal Poisson fibrered submanifold of $\left(M, \Lambda_{M}\right)$, then its normal bundle, with the induced Poisson structure, is also a fibrered Poisson manifold over $Q$.

Proof. For each $x \in P$ let $S_{M}(j(x))$ be the symplectic leaf of $\Lambda_{M}$ passing through the point $j(x)$, so that $\Lambda_{M \mid S(j(x))}$ is the inverse of a symplectic form $\omega_{M}(x)$ on $T_{j(x)} S(j(x))$ and $T_{j(x)}(j(P) \cap S(j(x)))^{\perp_{\omega_{M}}}$, the symplectic orthogonal of $T_{j(x)}(j(P) \cap S(j(x)))$, is a symplectic $r$ plane transversal to $T_{j(x)} j(P)$. The mapping of $M$ to $j(P)$ along the symplectic orthogonals gives a model of the normal bundle $\nu(P)$ of the embedding. Moreover, for each leaf $S_{P} \subset P$, the restriction of this model of normal bundle is the corresponding model for the embedding of that leaf $S_{P} \subset S_{M}$. In fact, one can choose any compatible almost-complex structure for the regular set of $\left(M, \Lambda_{M}\right)$ and consider the leafwise associated metric. $T_{j(x)}(j(P) \cap S(j(x)))^{\perp^{\perp \omega_{M}}}$ is then the orthogonal complement of $T_{j(x)}(j(P) \cap S(j(x)))$ with respect to this metric. We can use this leafwise metric to identify the normal bundle with a small enough tubular neighbourhood of $j(P)$. This open set inherits a Poisson structure which can be pulled back to the normal bundle (the Poisson structure depends on the almost-complex structure, but 
different choices give isomorphic structures). The local triviality of the fibre bundle $\tilde{\pi}: \nu(P) \rightarrow Q$ follows from that of the associated sphere bundle, which is a compact manifold (and the projection is a surjective submersion).

\section{The main construction: Poisson surgery}

Let $\left(M, \Lambda_{M}\right)$ be an $n$-dimensional Poisson manifold of rank $d$ and let $\left(P, \Lambda_{P}\right)$ be a fibrered compact $(n-2)$-dimensional Poisson manifold over the $(n-d)$-dimensional manifold $Q$ such that $\Lambda_{P}$ is compatible with the fibre bundle structure $\pi: P \rightarrow Q$. Suppose we have two disjoint embeddings $j_{a}: P \rightarrow M, a=1,2$, that both embed $\left(P, \Lambda_{P}\right)$ as a transversal Poisson fibrered submanifold of $\left(M, \Lambda_{M}\right)$. Assume that the normal bundles $\nu_{a}$ (using the model provided by Lemma 1 and considering the orientation induced by the Poisson bracket) have opposite Euler class. After identifying $\nu_{a}$ with a tubular neighbourhood $V_{a}$ of $j_{a}(P)$ by means of the maps $\hat{\mathrm{j}}_{a}: \nu_{a} \rightarrow V_{a}, a=1,2$, any orientation reversing identification $\psi: \nu_{1} \rightarrow \nu_{2}$ allows us to get a diffeomorphism $\varphi: V_{1}-j_{1}(P) \rightarrow V_{2}-j_{2}(P)$ preserving the orientation of the fibres (the disks) as the composition of $\psi$ with the diffeomorphism $h(x)=x /\|x\|^{2}$ that turns each punctured normal fibre inside out.

Definition 2. Let $\#_{\psi} M$ denote the smooth, foliated manifold, obtained from $M-\left(j_{1}(P) \cup j_{2}(P)\right)$ by identifying $V_{1}-j_{1}(P)$ with $V_{2}-j_{2}(P)$ via the composition $h \circ \psi$. If $M$ is a disjoint union $M_{1} \amalg M_{2}$ and $j_{a}$ maps $P$ into $M_{a}$, the manifold will be called the normal connected sum of $M_{1}$ and $M_{2}$ along $P$ (via $h \circ \psi$ ) and will be also denoted by $M_{1} \#_{\psi} M_{2}$.

It is easy to check that the diffeomorphism type of $\#_{\psi} M$ as a foliated manifold is determined by $\left(j_{1}, j_{2}\right)$ and the orientation reversing identification $\psi: \nu_{1} \rightarrow \nu_{2}$, up to fibre preserving isotopy. Once one of these identifications has been chosen, the remaining possibilities are $\left[P, S^{1}\right] \cong H^{1}(P ; \mathbb{Z})$.

4.1. Topological remarks. An orientation $\mu_{M}$ on $\left(M, \Lambda_{M}\right)$ determines, together with the Poisson structure, an orientation on $P$ in a neighbourhood of $j_{a}(P)$; this in turn, together with the restricted Poisson form $\omega_{P}$, determines an orientation on $Q$. It is clear that if the orientations on $Q$ obtained in this way from each neighbourhood $V_{a}$ are the same, then $\mu_{M}$ induces an orientation on $\#_{\psi} M$. 
There are some very well know results about the topology of $M_{1} \#_{\psi} M_{2}$ (see the remarks by Gompf [4]). First of all, $\#_{\psi} M$ is (oriented) cobordant to $M$. This is seen after identifying in the cobordism $M \times[0,1]$ neighbourhoods of $j_{1}(P)$ and $j_{2}(P)$ in the level $\{1\}$, and then rounding corners to get the cobordism manifold $X$. Hence, the Pontrjagin numbers $\left(\#_{\psi} M\right.$ oriented) behave additively, and in the even dimensional case the formuli for the Euler characteristic and signature are, respectively,

$$
\begin{gathered}
\chi\left(M_{1} \#_{\psi} M_{2}\right)=\chi\left(M_{1}\right)+\chi\left(M_{2}\right)-2 \chi(P) . \\
\sigma\left(M_{1} \#_{\psi} M_{2}\right)=\sigma\left(M_{1}\right)+\sigma\left(M_{2}\right),\left(\#_{\psi} M \text { oriented }\right)
\end{gathered}
$$

As is the case for symplectic manifolds, if $\#_{\psi} M$ is oriented the surgery construction is compatible (choosing an appropriate framing) with spin structures, and we can conclude:

Lemma 2. If $M$ admits a spin structure and $H^{2}(P ; \mathbb{Z})$ has no $\mathbb{Z}_{2}$ torsion, then there is a choice of $\psi$ such that $\#_{\psi} M$ admits a spin structure extending the one on $M$.

Proof. See [4], proposition 1.2.

4.2. Remarks regarding the foliation of $\#_{\psi} M$. If we start from a regular transversally orientable manifold $M$, then $\#_{\psi} M$ is also regular transversally orientable (orientability in regular Poisson manifolds is equivalent to transversal orientability) and its Godbillon-Vey class $G V\left(\# \psi_{\psi} M, \Lambda\right)$ can be computed in terms of the one of $M$. In particular:

Lemma 3. Let $M$ be transversally orientable. Then $G V\left(M, \Lambda_{M}\right)=0$ if and only if $G V\left(\#_{\psi} M, \Lambda\right)=0$.

Proof. We know that we can remove disjoint (closed) fibrered neighbourhoods $W_{a}$ of $j_{a}(P)$ so as to obtain an inclusion $i: M-\left(W_{1} \cup W_{2}\right) \rightarrow$ $\#_{\psi} M$ such that both ends of $M-\left(W_{1} \cup W_{2}\right)$ fibre over $P$ (with fibre an annulus). The condition $G V\left(M, \Lambda_{M}\right)=0$ implies, by naturality, the vanishing of the Godbillon-Vey class of $M-\left(W_{1} \cup W_{2}\right)$. Since its ends are fibrered, one can choose a representative $\beta$ of the class vanishing on these ends and deduce the existence of a form $\gamma$ vanishing on the ends whose exterior derivative is $\beta$. Finally, extending $\beta$ and $\gamma$ to forms $\tilde{\beta}$ and $\tilde{\gamma}$ defined on $M$ we obtain $d \tilde{\gamma}=\tilde{\beta}$, where $[\tilde{\beta}]=G V\left(M, \Lambda_{M}\right)$. The other direction is proven similarly. 
4.3. Constructing the Poisson form on $\#_{\psi} M$. Our final aim is to put a Poisson structure on $\#_{\psi} M$. To do that, we have to modify the previous construction slightly. Since we have to construct a symplectic structure on each resulting leaf, it is more convenient to use instead of the normal bundles, whose fibres have infinite area, the bundles $\nu_{a}^{0}$ of disks of radius $\pi^{-1 / 2}$. We will compose $\psi$ (which can be assumed to preserve the area of each fibre) with the map

$$
i(x)=\left(\frac{1}{\pi\|x\|^{2}}-1\right)^{1 / 2} x
$$

to turn each punctured disk inside out.

We notice that $V_{1}, V_{2}$ and $Y$, the image of $\left(V_{1} \cup V_{2}\right) \times[0,1]$ in $X$ (the cobordism between $M$ and $\left.\#_{\psi} M\right)$, are locally trivial fibre bundles over $Q$. Any closed form $\omega \in \Omega_{\text {fib }}^{k}\left(V_{1} \cup V_{2} \rightarrow Q\right)$ satisfying $j_{1}^{*} \omega=j_{2}^{*} \omega$ induces a form $\Omega_{\tilde{V}} \in \Omega_{\mathrm{fib}}^{k}(\tilde{V} \rightarrow Q)$, up to a choice of a compactly supported exact $k$-form $d \alpha, \alpha \in \Omega_{\text {fib }}^{k-1}(\tilde{V} \rightarrow Q)$, where $\tilde{V} \subset \#_{\psi} M$ is the image of $V_{1} \cup V_{2}$ in $\#_{\psi} M$. To get a representative $\Omega_{\tilde{V}}$ of this family we retrac disjoint neighbourhoods of $j_{a}(P)$ (containing $V_{a}^{0}$, the image of $\nu_{a}^{0}$ ) onto $j_{a}(P)$ and extend this map to a smooth retraction $\rho: M \rightarrow M$ isotopic to the identity. Then $\rho$ coincides with the identity outside a compact set of $V_{1} \cup V_{2}$, preserves the fibres of $V_{a}$, and commutes with $\hat{\mathrm{j}}_{2} \circ \psi \circ \hat{\mathrm{j}}_{1}^{-1}$ on $V_{1}$ and $V_{2}$. The $k$-form is the restriction to $\tilde{V}$ of the one induced on $Y$ by $\rho^{*} \omega$. Two different choices of the retraction will give rise to two $k$-forms whose difference will be a compactly supported element of $\Omega_{\mathrm{fib}}^{k}(\tilde{V} \rightarrow Q)$. To see that this compactly supported closed form is exact, it is enough to check it fibrewise. The procedure is the one described by Gompf. We recall that when we round corners to get the cobordism manifold $Y$, we can think of having added some new levels (i.e. we now have a map $p_{2}: Y \rightarrow[0,1+\epsilon]$ ) so that the level set $1+\epsilon$ is $\tilde{V}$, where the punctured disks of radius $\pi^{-1 / 2}$ are identified. As we go from $1+\epsilon$ to 1 we identify punctured disks of smaller and smaller radius until we reach the level set 1 where $j_{1}(P)$ and $j_{2}(P)$ are identified. The level sets corresponding to the values smaller than 1 are diffeomorphic to $V_{1} \cup V_{2}$. Given another retraction $\rho^{\prime}$, to evaluate the difference of the $k$-forms $\rho^{*} \omega_{\mid \tilde{V}}-\rho^{\prime *} \omega_{\mid \tilde{V}}$, we push down homotopically the corresponding (possibly singular) oriented $k$-manifold $M_{k} \subset p_{2}^{-1}(1+\epsilon]$ so that it lies in $p_{2}^{-1}([0,1])$ and the level 1 is contained in $j_{1}(P) \times\{1\}$. We then cut open $Y$ and project $\left(V_{1} \cup V_{2}\right) \times[0,1] \rightarrow V_{1} \cup V_{2} \times\{0\}$, and in the zero level set integrate $\rho^{*} \omega-\rho^{\prime *} \omega$ over the correspondent manifold with boundary 
$M_{k}^{\prime}$. But since the retractions were homotopic to the identity, both $\rho^{*} \omega$ and $\rho^{\prime *} \omega$ represent the same homology class as $\omega$. This, together with the fact that $j_{1}^{*} \omega=j_{2}^{*} \omega$, implies that $\int_{M_{k}^{\prime}} \rho^{*} \omega-\omega=0=\int_{M_{k}^{\prime}} \rho^{\prime *} \omega-\omega$. Hence their difference integrates to 0.

Now we will show that this construction works in the Poisson category.

Theorem 1. Let $\left(M, \Lambda_{M}\right)$ be an $n$-dimensional Poisson manifold of rank $d \geq 2$ and let $\left(P, \Lambda_{P}\right)$ be a compact $(n-2)$-dimensional Poisson manifold such that $\Lambda_{P}$ is compatible with the fibre bundle structure $\pi: P \rightarrow Q$, where $Q$ is an $(n-d)$-dimensional manifold. Let $j_{a}:\left(P, \Lambda_{P}\right) \rightarrow\left(M, \Lambda_{M}\right), a=1,2$, be two disjoint embeddings of $\left(P, \Lambda_{P}\right)$ as a transversal Poisson fibrered submanifold of $\left(M, \Lambda_{M}\right)$. Suppose that there is an orientation-reversing isomorphism of the normal bundles $\psi: \nu_{1} \rightarrow \nu_{2}$. Then $\#_{\psi} M$, the normal connected sum along the normal bundles of $j_{a}(P)$, can be given a canonical Poisson structure $\Lambda$, characterized as follows:

Suppose given disjoint identifications $\hat{\jmath}_{a}: \nu_{a} \rightarrow V_{a}$ of normal bundles with tubular neighbourhoods $V_{a}$ of $j_{a}(P)$ that send fibres into leaves. We denote by $\tilde{V}$ the image of $V_{1} \cup V_{2}$ in $\#{ }_{\psi} M$; then $\tilde{V}$ is a locally trivial fibre bundle with base space $Q$. Then there exists a unique fibre isotopy class of Poisson forms on $\tilde{V}$ containing elements $\omega$ satisfying the following characterization:

1) Let $\Omega_{\tilde{V}}$ be any of the 2 -forms induced in $\tilde{V}$ by $\omega_{M}$. Then the closed form $\omega-\Omega_{\tilde{V}} \in \Omega_{f i b}^{2}(\tilde{V} \rightarrow Q)$ has compact support and is exact.

2) The identification $\hat{\jmath}_{1}: \nu_{1} \rightarrow V_{1} \subset M$ can be chosen in such a way that the Poisson 2-form $\omega_{M}$ is $S O(2)$-invariant on $V_{1}^{0}=\hat{\jmath}_{1}\left(\nu_{1}^{0}\right)$, with $\nu_{1}^{0}$ the open disk bundle of radius $\pi^{-1 / 2}$, and on the closure of each fibre of $V_{1}^{0}$ it is symplectic with area $t_{0}$ independent of the fibre. Moreover, the forms $(1-s) \omega_{M}+s \pi^{*} \omega_{P}, 0 \leq s<1$, are all Poisson on the closure of $V_{1}^{0}$.

3) There is a closed vertical 2-form $\zeta$ with compact support in $V_{2}^{0}=$ $\hat{\jmath}_{2}\left(\nu_{2}^{0}\right)$, with $\nu_{2}^{0}$ the open disk bundle of radius $\pi^{-1 / 2}$, such that for all $t \in\left[0, t_{0}\right]$ the form $\omega_{M}+t \zeta$ is Poisson on both $V_{1} \cup V_{2}$ and $j_{2}(P)$.

4) There is a disk preserving map $\chi: \nu_{2} \rightarrow \nu_{2}$ isotopic to the identity by an isotopy with support in $\nu_{2}^{0}$, such that outside of a compact subset $K$ of $V_{1}^{0}$, the map $\varphi=\hat{\jmath}_{1} \circ \psi \circ i \circ \chi \circ \hat{\jmath}_{2}: V_{1}^{0}-j_{1}(P) \rightarrow V_{2}^{0}-$ $j_{2}(P)$ is Poisson with respect to the Poisson form $\tilde{\omega}_{M}=\omega_{M}+t_{0} \zeta$ 
on $M$. The manifold $\#_{\psi} M$ is obtained from $\left(M-\left(K \cup j_{2}(P)\right), \tilde{\omega}_{M}\right)$ by gluing via $\varphi$.

Moreover, different choices of embeddings of the normal bundles are connected by an isotopy that preserves the isotopy class described above.

Finally, the form $\omega$ depends smoothly on $\omega_{M}, \omega_{P}$ (and hence on $j_{1}, j_{2}$ ) and it can be constructed with each $V_{a}, a=1,2$ lying inside any preassigned neighbourhood of $j_{a}(P)$.

Now we will devote the next few paragraphs to proving Theorem 1.

4.4. The contraction operator. We recall that $\nu(P)$ is an $S O(2)$ bundle. Let $\tau_{s}: \nu(P) \rightarrow \nu(P), 0 \leq s \leq 1$, denote multiplication by $s$ on each disk and let $X_{s}$ denote the corresponding vector field. Since $X_{s}$ is a vertical vector field with respect to the fibre bundle structure $\nu(P) \rightarrow$ $Q$, we can define the operator $I: \Omega_{\mathrm{fib}}^{k}(\nu(P) \rightarrow Q) \rightarrow \Omega_{\mathrm{fib}}^{k-1}(\nu(P) \rightarrow Q)$ by

$$
I(\rho)=\int_{0}^{1} \tau_{s}^{*}\left(i_{X_{s}} \rho\right) d s .
$$

As usual, if $\rho$ is closed and $j^{*} \rho=0$, then $d I(\rho)=\rho$; moreover $I$ commutes with any action preserving the $S O(2)$-bundle structure.

Corollary 1. Let $\omega_{1}, \omega_{2}$ be two Poisson forms on $\nu(P)$ compatible with the fibre bundle structure $\nu(P) \rightarrow Q$ satisfying $j^{*} \omega_{1}=j^{*} \omega_{2}$ and inducing the same orientation on $\nu(P)$. There exist neighbourhoods $U_{1}, U_{2}$ of $P$ in $\nu(P)$ and an isomorphism $\phi: \nu(P) \rightarrow \nu(P)$ isotopic, relative to $P$, to the identity by an isotopy with compact support, such that $\phi: U_{1} \rightarrow U_{2}$ satisfies $\phi^{*} \omega_{2}=\omega_{1}$. If both forms already coincide over a compact subset $C$ of $P$, we may assume the isotopy to have support on a preassigned neighbourhood of the closure of $P-C$.

The isomorphism $\phi$ can be chosen to depend smoothly on $\omega_{1}$ and $\omega_{2}$. In fact, if we are given smooth families $\omega_{1, r}, \omega_{2, r}, b \leq r \leq c$, coinciding on a fixed neighbourhood of a given compact set $C$, and we construct isomorphisms $\phi_{b}, \phi_{c}$ as in the proof that follows satisfying $\phi_{b}^{*} \omega_{2, b}=\omega_{1, b}$ and $\phi_{c}^{*} \omega_{2, c}=\omega_{1, c}$, then there exists a smooth family $\phi_{r}$ such that $\phi_{r}^{*} \omega_{2, r}=\omega_{1, r}$ on a fixed neighbourhood of $P$ and $\phi_{r}$ equals the identity in the chosen neighbourhood of the closure of $P-C$. 
Proof. As in the proof of the Darboux-Weinstein theorem, we consider the vertical closed 2-form $\eta=\omega_{1}-\omega_{0}$ and the family $\omega_{t}=\omega_{0}+$ $t \eta$ (consisting also of vertical closed 2-forms). We can find a small neighbourhood of $P$ in which the $\omega_{t}$ are non-degenerate (because on $P$ both forms $\omega_{0}$ and $\omega_{1}$ induce the same orientation on the normal disk and because of the compactness of $P$ ). There, we know that $\eta=d \alpha$, with $\alpha=I(\eta)$, and we can find a family of vertical vector fields $Y_{t}$ characterized by the equation $i_{Y_{t}} \omega_{t}=-\alpha$. After using a suitable bump function, this 1-parameter family defines a global flow $\Psi_{t}$ on $\nu(P)$, leaving $P$ stationary. Computing $\frac{d}{d t}\left(\Psi_{t}^{*} \omega_{t}\right)$ we conclude that $\Psi_{t}^{*} \omega_{t}$ does not depend on $t$ near $P$. If the forms coincide in a neighbourhood of $C, \eta$ vanishes on that neighbourhood. For families the proof is trivial observing that the bump functions chosen at the ends $b, c$ can be smoothly joined.

Corollary 2. Let $\left(M, \Lambda_{M}\right)$ be an $n$-dimensional Poisson manifold of rankd. Let $\left(P, \Lambda_{P}\right)$ be a regular compact Poisson manifold of dimension $n-2$ which fibres over the $(n-d)$-dimensional manifold $Q$ and such that $\Lambda_{P}$ is compatible with the fibration. Assume that $j_{a}:\left(P, \Lambda_{P}\right) \rightarrow$ $\left(M, \Lambda_{M}\right), a=1,2$, embeds $\left(P, \Lambda_{P}\right)$ as a transversal Poisson fibrered submanifold of $\left(M, \Lambda_{M}\right)$. Suppose that both normal bundles are trivial and let $\psi: \nu_{1}(P) \rightarrow \nu_{2}(P)$ be a bundle isomorphism identifying them and preserving the orientation of the fibres. Then $\#_{\psi} M$ can be given a Poisson structure $\Lambda$.

Proof. We can identify each normal bundle with $P \times \mathbb{R}^{2}$ in such a way that each disk $\{z\} \times D^{2}$ has area form $d x \wedge d y$. So we have isomorphisms $\hat{\mathrm{\jmath}}_{a}: P \times D_{\epsilon}^{2} \rightarrow V_{a}, a=1,2$, and $\tilde{\psi}: P \times D_{\epsilon}^{2} \rightarrow P \times D_{\epsilon}^{2}$. The main point is that since the normal bundles are trivial, $j_{a}^{*} \omega_{a}+d x \wedge d y$ are Poisson structures that restrict to $j_{a}^{*} \omega_{a}$ on $P$. Hence, we can find a real number $\delta>0$, and diffeomorphisms $\tilde{\mathrm{j}}_{a}: P \times D_{\delta}^{2} \rightarrow U_{a}$ with $\tilde{\mathrm{j}}_{1}^{*} \omega_{1}=j_{1}^{*} \omega_{1}+$ $d x \wedge d y, \tilde{\psi}^{*} \tilde{\mathrm{j}}_{2}^{*} \omega_{2}=j_{1}^{*} \omega_{1}+d x \wedge d y, U_{a} \subset V_{a}$ neighbourhoods of $j_{a}(P)$. Composing $\tilde{\psi}$ with the area preserving map $(r, \theta) \mapsto\left(\sqrt{\delta^{2}-r^{2}},-\theta\right)$ preserves the Poisson structure and hence allows us to define a Poisson structure on $\#_{\psi} M$. We notice that we could have equally asked our initial fibre bundle morphism $\psi$ to reverse the orientation of the fibres, because by composing with the leafwise reflection $(r, \theta) \mapsto(r,-\theta)$ one can always reverse the orientation of a trivial bundle.

In the above construction, the Poisson structure coincides with $\Lambda_{M}$ on $M-\left(j_{1}(P) \cup j_{2}(P)\right)$. But we have to allow perturbations in a 
neighbourhood of one of the embeddings to have uniqueness up to isotopy.

The main obstruction to finally solving the problem posed in Theorem 1 in general is that one cannot put a global Poisson structure on $\nu_{a}$ induced by $j_{a}^{*} \omega_{a}$ and the symplectic structure on the symplectic orthogonal, unless the normal bundle is trivial. We can overcome this difficulty in the following way. We consider $\nu_{a}^{0}$, the bundles of open disks of radius $\pi^{-1 / 2}$, and identify the punctured disks by composing $i$ with $\psi$ to get $\mathcal{B}$, an $S^{2}$-bundle with structural group $S O(2)$ whose fibres have an $S O(2)$-invariant area form $\omega_{S^{2}}$ that integrates to 1 on each of them. We have two embeddings $i_{0}: P \rightarrow \mathcal{B}, i_{\infty}: P \rightarrow \mathcal{B}$ with $\hat{\mathrm{j}}_{1} \circ i_{0}=j_{1}, \hat{\mathrm{j}}_{2} \circ i_{\infty}=j_{2}$. Let us put $E^{0}=\mathcal{B}-P_{\infty}\left(\operatorname{resp} . E^{\infty}=\mathcal{B}-P_{0}\right)$. Using Thurston's ideas (see [9], Thm. 6.3) we can construct a vertical 2-form $\eta$ restricting to the above defined area form on each fibre. We consider a form $\beta$ on $q: \mathcal{B} \rightarrow P$ representing the Poincaré dual of $P_{0}$ so that it integrates to 1 on each fibre (sphere transverse to $P_{0}$ ). It can be chosen to have support in a small neighbourhood of $P_{0}$, so that it vanishes on $P_{\infty}$. We take trivializations $h_{k}: q^{-1}\left(\mathcal{U}_{k}\right) \rightarrow \mathcal{U}_{k} \times S^{2}$ of $\mathcal{B}$ and a partition of unity $\rho_{k}$ subordinate to $\left\{\mathcal{U}_{k}\right\}$. Since $h_{k}^{*} \pi_{S^{2}}^{*} \omega_{S^{2}}-\beta=d \alpha_{k}$ on $q^{-1}\left(\mathcal{U}_{k}\right), \eta=f\left(\beta+d \sum_{k}\left(\rho_{k} \circ q\right) \alpha_{k}\right)$, where $f$ is the forgetful map $f: \Omega^{2}(\mathcal{B}) \rightarrow \Omega_{\mathrm{fib}}^{2}(\mathcal{B} \rightarrow Q)$, satisfies the requirements. The result of averaging $\eta-q^{*} i_{0}^{*} \eta$ (both $q, i_{0}$ are maps lifting id: $Q \rightarrow Q$ ) under the $S O(2)$-action is a vertical $S O(2)$-invariant 2 -form, that we will still call $\eta$, that restricts to the canonical volume form on each sphere and satisfies $i_{0}^{*} \eta=0$. We can even choose $\eta$ so that $\eta_{\mid E^{0}}$ extends over $\nu_{1}$ to a closed vertical form that is symplectic on the planes (fibres). We only need to pick $\beta$ with support away of $P_{\infty}$, so that on the intersection of that neighbourhood with $q^{-1}\left(\mathcal{U}_{i}\right)\left(\mathcal{U}_{i}\right.$ contractible) $\alpha_{k}$ can be chosen to be $h_{k}^{*} \pi_{S^{2}}^{*} \alpha^{\prime}$, for any $\alpha^{\prime}$ with $d \alpha^{\prime}=\omega_{S^{2}}$ on that neighbourhood. In particular, the restriction of the 1 -form $\alpha=1 / 2\left(r^{2}-\frac{1}{\pi}\right) d \theta \in \Omega^{1}\left(\mathbb{R}^{2}-\{0\}\right)$ (given in polar coordinates) to the disk of radius $\pi^{-1 / 2}$ admits an extension to a form $\alpha^{\prime}$ on $S^{2}-\{0\}$ with $d \alpha^{\prime}=\omega_{S^{2}}$.

The forms $\omega_{t}=q^{*} j_{1}^{*} \omega_{1}+t \eta$ are non-degenerate for $0<t \leq t_{1}$ because, as Thurston observed, $q^{*} j_{1}^{*} \omega_{1}$ is non-degenerate on the orthogonal to the tangent space of the spheres (which does not depend on $t$ because it is determined by $\eta$ ), and for a choice of $\eta$ extending to $\nu_{1}$ as described above, the forms $\omega_{t}$ will be symplectic near the closure of $E^{0} \cong \nu_{i}^{0}$ in $\nu_{1}$ for $t_{1} \leq t$ small enough. 
4.5. Comparing the Poisson structures on $\mathcal{B}, E^{0}$ and $E^{\infty}$. Now that we have a family of closed non-degenerate 2 -forms on $\mathcal{B}$, we would like to compare one of them with the ones defined on $E^{0} \cong \nu_{1}^{0}$ and $E^{\infty} \cong \nu_{2}^{0}$ that come from $\omega_{1}$ and $\omega_{2}$. Following Corollary 1, for each $t$ we could find neighbourhoods $\left(\mathcal{W}_{0}^{t}, \omega_{t}\right)$ of $P_{0}$ and $\left(\mathcal{W}_{\infty}^{t}, \omega_{t}\right)$ of $P_{\infty}$ which are Poisson equivalent to some neighbourhoods (depending on $t$ ) of $\left(j_{1}(P), \omega_{1}\right)$ and $\left(j_{2}(P), \omega_{2}\right)$. But nothing guarantees that $\mathcal{B}=\mathcal{W}_{0}^{t} \cup \mathcal{W}_{\infty}^{t}$, for some $t$.

Instead, we use Gompf's construction again. On $E^{0}$, let $\varphi=I(\eta)$ and define the vertical vector fields $Y_{t}, 0<t \leq t_{1}$ by the condition $i_{Y_{t}} \omega_{t}=-\varphi$ (also defined in a neighbourhood of the closure of $E^{0}$, if $\eta$ was chosen to extend to $\left.\nu_{1}\right)$. The key property is that these vector fields are $S O(2)$-invariant. For a fixed $t_{0}$, the flow $\Psi_{t}$, required to be the identity for $t=t_{0}$, is $S O(2)$-invariant and of course satisfies $\Psi_{t}^{*} \omega_{t}=\omega_{t_{0}}$. In principle, we know that for any $S O(2)$-invariant compact set $K \in E^{0}$ there exists an interval $J$ of $t_{0}$ in $\left(0, t_{1}\right]$ where the flow $\Psi: K \times J \rightarrow E^{0}$ is defined. But in can be shown that $\Psi$ is defined on $E^{0} \times\left[t_{0}, t_{1}\right]$. Given any point $x$ on $E^{0}$, the action $\Psi$ determines an $S O(2)$-orbit on its fibre and hence a disk $D(x)$. We define:

$$
A(x)=\int_{D(x)} \eta
$$

and

$$
A_{t}(x)=\int_{D(x)} \omega_{t},
$$

where the forms are pulled back to the disk. The map $A: E^{0} \rightarrow[0,1)$ is a smooth $S O(2)$-invariant proper surjection and it is clear that $A_{t}(x)=$ $t A(x)$. Given $x \in E^{0}, t_{0} \in\left(0, t_{1}\right]$ and $K=D(x)$ we obtain a flow as above on $D(x)$. Let $D\left(\Psi_{t}(x)\right)$ be the disk whose boundary is the $S O(2)$-orbit of $\Psi_{t}(x)$ (it is also $\Psi_{t}(\partial D(x))$ ). Then we have:

$$
\begin{aligned}
t A\left(\Psi_{t}(x)\right) & =A_{t}\left(\left(\Psi_{t}(x)\right)\right)=\int_{D\left(\Psi_{t}(x)\right)} \omega_{t} \\
& =\int_{\Psi_{t}(D(x))} \omega_{t}=\int_{D(x)} \Psi_{t}^{*} \omega_{t}=\int_{D(x)} \Psi_{t_{0}}^{*} \omega_{t_{0}}=t_{0} A(x),
\end{aligned}
$$

So we can conclude that $A\left(\Psi_{t}(x)\right)=\frac{t_{0}}{t} A(x)$. Since $A$, which is proper, decreases with the flow lines (with $t$ increasing), these flow lines cannot leave $E^{0}$ and hence $\Psi$ is defined in $E^{0} \times\left[t_{0}, t_{1}\right]$. The inequality $A\left(\Psi_{t_{1}}(x)\right)<\frac{t_{0}}{t_{1}}$ implies that choosing $t_{0}$ small enough, $\Psi_{t_{1}}$ sends $E^{0}$ 
into any initially fixed tubular neighbourhood of $P_{0}$. In particular, we choose $t_{0}$ so that $\Psi_{t_{1}}\left(E^{0}\right) \subset \mathcal{W}_{0}^{t_{1}}$. Hence $\hat{\jmath}_{1} \Psi_{t_{1}}$ sends $\left(E^{0}, \omega_{t_{0}}\right)$ into $\left(\hat{\mathrm{j}}_{1} \Psi_{t_{1}}\left(E^{0}\right), \omega_{1}\right)$. Actually, the Poisson morphism $\Psi_{t_{1}}$ extends to a neighbourhood of the closure of $E^{0}$, for suitably chosen $\eta$ and thus it can further be extended to a diffeomorphism $\Psi_{t_{1}}: \nu_{1} \rightarrow \nu_{1}$ isotopic to the identity by an isotopy with compact support (but Poisson only in a neighbourhood of the closure of $\left.E^{0} \cong \nu_{1}^{0}\right)$.

The restriction of each $\omega_{t}$ to $E^{\infty}$ also induces a Poisson structure, but in general $i_{\infty}^{*} \omega_{t} \neq j_{2}^{*} \omega_{2}$. But we can modify $\omega_{2}$ in a neighbourhood of $j_{2}(P)\left(\omega_{2}\right.$ has not been involved in any of the previous work) so that the above equality holds. We choose an $S O(2)$-equivariant map $\mu: \mathcal{B} \rightarrow \mathcal{B}$ lifting $i d: P \rightarrow P$ such that $\mu$ fixes a neighbourhood of $P_{\infty}$ and collapses a neighbourhood of $P_{0}$ to $P_{0}$. The composition of the restriction of $\hat{\mathrm{j}}_{2}^{-1}$ to $V_{2}^{0}$ with $\mu$ can be extended to a map $\lambda$ from a closed neighbourhood $U_{2}$ of $V_{2}^{0}$ in $V_{2}$ (a neighbourhood of $\partial U_{2}$ is sent to $\left.P_{0}\right)$. We can then modify the Poisson structure of $\left(U_{2}, \omega_{2}\right) \subset\left(V_{2}, \omega_{2}\right)$, without modifying the symplectic foliation, by adding to $\omega_{2}$ a closed vertical 2 -form $\zeta$ such that $\omega_{2}+\zeta$ is non degenerate (and hence Poisson) and $\zeta$ vanishes in a neighbourhood of $\partial U_{2}$ in $U_{2}$. We set $\zeta=\lambda^{*} \eta$. By the compactness of $P$ there exists $t_{2}>0$ such that for all $0 \leq t \leq t_{2}$, $\tilde{\omega}_{M}=\omega_{2}+t \zeta$ is non degenerate. To solve the problem we just need to pick our previous $t_{0}$ smaller than $t_{2}$ (and use of course $\tilde{\omega}_{M}=\omega_{M}+t_{0} \zeta$ ). So by gluing we can define a Poisson form $\omega$ on $\tilde{V}$ that satisfies all the requirements of Theorem 1 . To be more precise, we can find a map $\chi: E^{\infty} \rightarrow E^{\infty}$ isotopic to the identity by an isotopy (relative to $P_{\infty}$ ) with support in $\nu_{2}^{0}$ and Poisson, with respect to the forms $\omega_{t_{0}}$ and $\omega_{M}+t_{0} \zeta$, in a neighbourhood $U_{\infty}$ of $P_{\infty}$; the map can actually be extended to a diffeomorphism of $\nu_{2}$ isotopic to the identity. We glue using the map $\hat{\mathrm{j}}_{2} \circ \chi \circ i \circ \psi \circ \Psi_{t_{1}}^{-1} \circ \hat{\mathrm{j}}_{1}^{-1}: V_{1}^{0} \rightarrow V_{2}^{0}$, where $\Psi_{t_{1}}$ and $\chi$ are thought as diffeomorphisms of the normal bundles (instead of having domain in the sphere bundle $\mathcal{B}$ ). The embeddings we finally use are $\hat{\mathrm{j}}_{1} \circ \Psi_{t_{1}}$ and modified $\hat{\mathrm{j}}_{2}$ by composing on the right with $\chi: \nu_{2} \rightarrow \nu_{2}$. The only condition that needs to be checked is that the difference $\left[\omega-\Omega_{\tilde{V}}\right]$ (which by construction has compact support) is exact. As we saw above, it is enough to check this fibrewise. Thus, it is enough to show that

$$
\left\langle\omega-\Omega_{\tilde{V}}, F\right\rangle=0, \forall F \in H^{2}(\tilde{N}, \mathbb{Z})
$$


for all the fibres $\tilde{N}$ of $\tilde{V} \rightarrow Q$. This time we will not write the proof of equation 6 because it is, word for word, Gompf's proof ([4] pages 547-548).

Concerning uniqueness, for any smooth family of Poisson forms $\omega_{t} \in H_{\text {fib }}^{2}(\tilde{V} \rightarrow Q), t \in[0,1]$ such that the forms $\omega_{t}-\Omega_{\tilde{V}}$ are exact and compactly supported, the forms $\omega_{t}-\omega_{0}$ are exact in compactly supported cohomology (we can find a common compact set $W$ of $\tilde{V}$ containing all the supports). Hence we can find a family of compactly supported 1-forms $\alpha_{t}$ with $\frac{d}{d t} \omega_{t}=\frac{d}{d t}\left(\omega_{t}-\omega_{0}\right)=d \alpha_{t}$ and apply Moser's theorem to show that there is an isotopy with support in $W \subset \tilde{V}$ pulling back all the forms of the family to $\omega_{0}$. The isotopy class of the form constructed using the procedure just described is fixed. A different choice of $t \leq t_{0}$ can be absorbed by using the parametrized version of corollary 1. Equally, for any other choice $\hat{\eta}$ the family $\eta_{s}=s \eta+(1-s) \hat{\eta}$ is valid for the construction and we can again apply the same corollary to the family $\Psi_{s, t}$. Any other choices can be connected by smooth families, and the same happens when we change the embeddings of the normal bundles (preserving the foliations) and the choice of $\psi$ (preserving the fibre bundle structure) by isotopic identifications.

Any Poisson 2-form $\omega$ satisfying the four conditions of theorem 1 is isotopic to one constructed using the procedure described above. We use $\psi$ to recover the sphere bundle $\mathcal{B}$ and the modified embeddings to put in $\mathcal{B}$ a $S O(2)$-invariant Poisson form $\omega_{t_{0}}$ that agrees with $\omega_{M}$ on $V_{1}^{0}$ and with $\tilde{\omega}_{M}$ near $j_{2}(P)$. This is also the result of applying the construction of the theorem with $\eta=\frac{1}{t_{0}}\left(\omega_{t_{0}}-q^{*} \omega_{P}\right)$ and $t_{1}=t_{0}$. $\mathrm{SO}(2)$-invariance implies that the fibres are $\omega_{t_{0}}$-orthogonal to $P_{\infty}$, so $\eta$ is actually non-degenerate on the fibres at $P_{\infty}$. Non degeneracy of $\omega_{t}$ at $P_{\infty}, t \leq t_{0}$, follows from condition 3 , applied first to $T P_{\infty}$. We can extend $\eta$ to $\nu_{1}$ after shrinking the embedding $\hat{\mathrm{j}}_{1}: \nu_{1} \rightarrow M$ relative to $E^{0}$ (non-degeneracy is an open condition). If we apply the construction to the embedding of condition 2 ( $\operatorname{shrunk}$ (relative to $E^{0}$ ), if necessary), when $t=t_{0}$ we get the same embedding $\left(\Psi_{t_{0}}=i d\right)$ because it was already Poisson. The same happens for the second embedding (the correction $\chi$ equals the identity), provided we chose the given $\zeta$ defining $\tilde{\omega}_{M}$, rather than setting $\zeta=\lambda^{*} \eta$. Hence, the gluing map equals $\varphi^{-1}$ near $j_{2}(P)$. The only price to pay is that $\zeta$ may not be $\lambda^{*} \eta$, for $\lambda$ extending the restriction of $\hat{\mathrm{j}}_{2}^{-1}$ to $V_{2}^{0}$ (but we have that $j_{2}^{*} \zeta=i_{\infty} \eta$, and $\zeta$ can be assumed to vanish outside $\left.\hat{\mathrm{j}}_{2}\left(\mathcal{B}-P_{0}\right)=V_{2}^{0}\right)$. We will show 
that $\omega$ and $\omega^{\prime}$, constructed using $\zeta^{\prime}=\lambda^{*} \eta$, are isotopic by an isotopy fixing the complementary of a compact set in $\tilde{V}$. It will be enough to show that the the Poisson forms constructed using $\zeta_{s}=s \zeta^{\prime}+(1-s) \zeta$ satisfy the condition 1 of the theorem. But that can be proven using the ideas that proved $\left\langle\Omega_{\tilde{V}}-\omega, F\right\rangle=0 \forall F \in H^{2}(\tilde{N}, \mathbb{Z})$ (see $[4]$ page 549).

\section{The modular class of $\#_{\psi} M$}

Let $\left(M, \Lambda_{M}\right)$ be a Poisson manifold; we assume for simplicity that $M$ is orientable. One of the first invariants of the Poisson structure one has is the modular class [12]. Roughly speaking, the modular class measures to what extent the Poisson manifold admits a transverse measure (transverse to the leaves) invariant by all the hamiltonian vector fields. The modular class belongs to the first Poisson cohomology group of $\left(M, \Lambda_{M}\right)$ (see $\left.[\mathbf{1 0}]\right)$. For each volume form $\mu$, a vector field representing the modular class is defined by the formula

$$
\phi_{\mu}: f \mapsto \operatorname{div}_{\mu} X_{f},
$$

where $X_{f}$ is the Hamiltonian vector field associated to $f$ and $d i v_{\mu}$ the divergence with respect to $\mu$.

A Poisson manifold with vanishing modular class is called unimodular. It is clear from what we said above that an orientable Poisson manifold is unimodular if and only if there exists a volume form invariant by all the hamiltonian vector fields. Since (at least in the regular set) a volume form is the wedge product of the leafwise Liouville volume form (which is invariant by the hamiltonian vector fields) and a transverse volume form, the invariance of this transverse volume form is equivalent to the invariance of the whole form (which is why we spoke about measuring the existence of a invariant transverse volume form).

Now let us assume that $\#_{\psi} M$ is oriented.

Proposition 2. If $\left(\# \psi_{\psi} M, \Lambda\right)$ is unimodular then $\left(M, \Lambda_{M}\right)$ is also unimodular, but the converse is not true.

Proof. We first notice that if we have an oriented Poisson manifold $\left(N, \Lambda_{N}\right)$ and an open set $U$ such that $\left(U, \Lambda_{N \mid U}\right)$ is unimodular, then $\left(N, \Lambda_{N}\right)$ will be unimodular if any of the invariant volumes on $\left(U, \Lambda_{N \mid U}\right)$ can be extended to an invariant volume on $\left(N, \Lambda_{N}\right)$. We will see that there are cases where $\left(N, \Lambda_{N}\right)$ is unimodular but not all the 
invariant volumes on a certain open set can be extended to be invariant on $\left(N, \Lambda_{N}\right)$. It is worth noticing that when $\left(N, \Lambda_{N}\right)$ is a Poisson fibrered manifold and $U$ cuts each leaf in a non-empty open connected set, then any invariant volume form in $\left(U, \Lambda_{N \mid U}\right)$ extends to a unique invariant form on $\left(N, \Lambda_{N}\right)$ [12]. It follows easily that in a general Poisson manifold $\left(N, \Lambda_{N}\right)$, if we take a closed set $V$ contained in a connected open one $U$, such that $U$ is fibrered and $V$ intersects each fibre in a non-empty set whose complement (in the fibre) is connected, then $\left(N, \Lambda_{N}\right)$ is unimodular if and only if $\left(N-V, \Lambda_{N \mid N-V}\right)$ is unimodular. As a consequence, any perturbation of the Poisson bivector on $V$ that preserves the foliation does not affect the unimodularity (resp. non-unimodularity) of $\left(N, \Lambda_{N}\right)$. Hence, the unimodularity of $\left(\#{ }_{\psi} M, \Lambda\right)$ implies the unimodularity of $\left(M, \Lambda_{M}\right)$. If we start with $\left(M, \Lambda_{M}\right)$ unimodular, since $V_{a}$ fibres over $Q$, any invariant volume on $\left(M, \Lambda_{M}\right)$ will determine a couple of volume forms on $Q$. It is clear that $(\#, M, \Lambda)$ will be unimodular if and only if we are able to find an invariant volume form such that the induced volume forms on $Q$ are the same. Though in general this not true (and we will end the proof of the proposition by constructing counterexamples), we will describe now some situations where it does occur.

Definition 3. Take $\left(M, \Lambda_{M}\right),\left(P, \Lambda_{P}\right)$ and $j_{1}:\left(P, \Lambda_{P}\right) \rightarrow\left(M, \Lambda_{M}\right)$ as in theorem 1. Assume that $j_{1}(P)$ has trivial normal bundle. Then once we have fixed a trivialization $\psi$ of the normal bundle, we can apply our construction to the disjoint union of $\left(M, \Lambda_{M}\right)$ with $\left(M, \Lambda_{M}\right)$. We denote the resulting manifold by $\left(M \#{ }_{\psi} M, \Lambda_{M} \# \Lambda_{M}\right)$

Corollary 3. Let $\left(M, \Lambda_{M}\right),\left(P, \Lambda_{P}\right)$ be as in the above definition. Then $\left(M, \Lambda_{M}\right)$ is unimodular if and only if $\left(M \#{ }_{\psi} M, \Lambda_{M} \# \Lambda_{M}\right)$ is unimodular.

To construct counterexamples we begin by proving the following lemma:

Lemma 4. There exist Poisson fibrered manifolds (actually symplectic bundles) with open sets having invariant volume forms which do not extend to invariant volume forms on the whole manifold.

Proof. The idea is to start with our fibrered open set, and then glue some of the fibres into a single one (so we are putting restrictions on the volume form we pull back from the base space). We consider the Poisson fibrered manifold $S^{2 n-1} \times D^{2} \rightarrow S^{2 n-1}$, where $D^{2}$ is the 
corresponding closed unit disk with its usual symplectic form (have in mind the case $n=1$ ). For each point of $S^{2 n-1}$ we consider its image by the antipodal map and identify the boundaries of the corresponding fibres via a reflection (say, on the $y$-axis) $r_{y}: S^{1} \rightarrow S^{1}$. The resulting manifold is a symplectic bundle over $\mathbb{R} P^{2 n-1}$ with fibre the sphere with the usual area form (it can also be constructed by considering $S^{2 n-1} \subset$ $\mathbb{R}^{2 n} \subset \mathbb{R}^{2 n+1}$, taking a closed tubular neighbourhood of fixed radius of $S^{2 n-1} \subset \mathbb{R}^{2 n+1}$ and identifying its boundary using the antipodal map and then rescaling the area form). If we remove all the equators we obtain the initial open disk bundle. In this open set, the invariant volume forms come from volume forms on $S^{2 n-1}$, but only the ones invariant under the action of the antipodal map on $S^{2 n-1}$ extend to invariant volume forms on the whole manifold.

There is a third way of constructing these manifolds, starting from the final Poisson manifold, which gives many more examples. We choose $(Q, G,(F, \omega), \rho)$ where $Q$ is a compact manifold, $G$ is a normal subgroup of $\pi_{1}(Q)$ of finite index and $\rho$ is a representation of $K=\pi_{1}(Q) / G$ in the group of symplectomorphisms of $(F, \omega)$ such that there are points in $F$ with trivial stabilizers. $Q_{G}$, the cover of $Q$ associated to the $\operatorname{subgroup} G$ is a principal $K$-bundle, so we can construct the associated bundle to the chosen representation $\rho$ by symplectomorphisms. Our resulting manifold $M$ is a symplectic bundle and hence a Poisson manifold, but as a bundle, since it has discrete structural group, it has the unique lifting property. Thus, if on the fibre over the base point $x_{0}$ of $Q$, we pick a point $z$ with trivial stabilizer, the lifting to $z$ of all the homotopy classes of paths based on $x_{0}$ gives us an embedding of $Q_{G}$ in $M$ transverse to the fibres. On the fibre over $x_{0}$, the points close to $z$ have trivial stabilizer which implies that the normal bundle to $Q_{G}$ is trivial. We can even take as a tubular neighbourhood the result of pushing a small disk around $z$ using the unique lifting property which gives us a symplectic subbundle. It is clear that the invariant volumes on a small tubular neighbourhood of $Q_{G}$ that extend to invariant volume forms on the whole manifold are those which come from $K$-invariant volume forms on $Q_{G}$.

Now we are ready to finish the proof of proposition 2 :

To construct the counterexample we take two copies of any of the symplectic fibrations $(Q, G,(F, \omega), \rho) \rightarrow Q$ of lemma 4 (with $F$ a surface) and consider in both the same embedding of $Q_{G}$. Next we fix a volume form $\mu$ on $Q_{G}$ that descends to $Q$. Then we pick a point 
$z \in Q_{G}$ and consider a diffeomorphism $f: Q_{G} \rightarrow Q_{G}$ homotopic to the identity (relative to $z$ ) which is the identity in a neighbourhood of the remaining points of the orbit of $z$ and which does not preserve $\mu$ in $z$. We identify both embeddings of $Q_{G}$ in $M$ via $f$ and perform the fibrered connected sum using any framing $\psi$ to obtain a manifold $\left(M \#{ }_{\psi} M, \Lambda_{M} \# \Lambda_{M}\right)$ which is non unimodular. If it were, an invariant volume form would induce a volume form $e^{f} \mu$ on $Q_{G}$ both invariant by the action of $K$ and the action of $K$ conjugated by $f$, but this cannot happen at the point $z$.

\section{Poisson manifolds with arbitrary fundamental groups}

Using the previous results we can prove the theorem that extends Gompf's existence theorem to regular Poisson manifolds.

Theorem 2. Let $G$ be any finitely presentable group. Then for any integers $n \geq d \geq 4$, $d$ even, there exist an oriented closed regular Poisson manifold of dimension $n$ and constant rank $d\left(M^{n, d}, \Lambda\right)$ such that $\pi_{1}\left(M^{n, d}\right) \cong G$. These manifolds have vanishing Godbillon-Vey class but the ones with a codimension one symplectic foliation are nonunimodular. Moreover, they can be chosen to be spin.

Proof. As we remarked in the introduction, we only need to prove the case $n=5, d=4$ because Gompf already established the result for all cases except for $n$ odd and $d=n-1$, and the odd higher dimensional cases follow from the 5-dimensional one (by multiplying by simply connected symplectic manifolds of the appropriate dimension in the case of a codimension one symplectic foliation).

We first recall Gompf's proof. He starts with a closed symplectic manifold $T^{2} \times \Sigma_{g}$ such that $G$ can be obtained by collapsing some elements of its fundamental group. The symplectic form is chosen so that these elements are the simple curves of some trivially embedded symplectic tori. The key step is that the manifold which is glued along each of these tori is a rational elliptic surface (along one of its regular fibres), and the resulting fundamental group, which does not depend on the chosen framing, is the old one with the homotopy of these tori killed. It is worth recalling the topology of these rational elliptic surfaces. They are diffeomorphic to $\mathbb{C} P^{2} \stackrel{9}{\#}\left(-\mathbb{C} P^{2}\right)$ and an example can be constructed by blowing up the nine points of $\mathbb{C} P^{2}$ where two generic cubics intersect. We get in this way a fibration $p: \mathbb{C} P^{2} \stackrel{9}{\#}\left(-\mathbb{C} P^{2}\right) \rightarrow$ 
$\mathbb{C} P^{1}$ whose fibres are the pencil of cubics generated by the two given ones. The general fibre is a smooth cubic (topologically a torus) and we also have 12 singular fibres each of which is topologically a sphere with a self intersection point (the result of collapsing a nonseparating regular curve of the generic fibre). It is easy to check that the complement of a regular fibre is simply connected. Roughly speaking, the complement fibres over a disk so we only have to worry about the fibre. Following [7], we see that this complement can be constructed starting from $D^{2} \times$ $T^{2}, T^{2}=\langle a\rangle \times\langle b\rangle$, and extending the fibration to a bigger disk (in $\mathbb{C} P^{1}$ ) containing a singular fibre amounts to gluing a two handle (with some framing) over either $a$ or $b$ (we have 12 singular fibres, and 6 of the disks go over $a$ and 6 over $b$ ). The last step is to glue a neighbourhood of the regular fibre over $\infty$. Hence, any curve contained in a fibre is trivial in $p^{-1}\left(\mathbb{C} P^{1}-\{0, \infty\}\right)$.

To get our Poisson 5-manifold $M$ with $\pi_{1}(M) \cong G$, we consider one of Gompf's manifolds $\left(M_{G}, \omega_{M_{G}}\right)$ with $\pi_{1}\left(M_{G}\right)=G$. We can also assume that $M_{G}=N_{G} \# \mathbb{C} P^{2} \stackrel{9}{\#}\left(-\mathbb{C} P^{2}\right)$ and that the fibre removed is $p^{-1}(\infty)$. Let $M_{1}=M_{G} \times S^{1}$ with the product Poisson structure (the vertical 2-form $p_{1}^{*} \omega_{M_{G}}$, which we rename $\omega_{M_{G}}$ ). In $M_{G}$, the fibre $p^{-1}(0)=T$ is a trivially embedded symplectic torus with symplectic form $\omega_{0}$. Now let $M_{2}=T \times S^{3}$ with the product Poisson structure coming from $\omega_{0}$ and a Poisson structure of $S^{3}$ determined by the Reeb foliation and the usual volume form, and let $k \subset S^{3}$ be the unknot, which is a Poisson submanifold of $S^{3}$ transverse to the foliation. We consider the Poisson submanifolds $P_{1}=T \times S^{1} \subset M_{1}, P_{2}=T \times k \subset$ $M_{2}$. It is clear that both are transversal Poisson fibrered submanifolds. Moreover, they are trivially embedded and any identification of $k$ with the factor $S^{1}$ of $P_{1}$ identifies $P_{1}$ and $P_{2}$ as Poisson manifolds. Any identification between normal bundles will allow us to construct the corresponding connected sum along the normal directions. In this case, we have canonical framings; the one in $P_{1}$ comes from the projection $p: \mathbb{C} P^{2} \stackrel{9}{\#}\left(-\mathbb{C} P^{2}\right) \rightarrow \mathbb{C} P^{1}$ and the one in $P_{2}$ from the zero-framing of the unknot. Using this framing and $\langle a, b, s\rangle$ as base of $H_{3}(T \times$ $S^{1} ; \mathbb{Z}$ ) (the choice of $s$ depends on the orientation we pick for $M_{1}$ ), any other framing is given by a triple $\left(l_{1}, l_{2}, l_{3}\right) \in \mathbb{Z}^{3}$. We will denote the Poisson manifold so obtained by $M_{1} \#_{\left(l_{1}, l_{2}, l_{3}\right)} M_{2}$. The computation of its fundamental group is mere routine, but we will do it anyway because this is not quite the manifold we are looking for. As usual, we apply Seifert-Van Kampen's theorem: 
Let $D_{1}$ be the unit disk contained in $\mathbb{C P}^{1}$ and $W_{2}=k \times D_{2}$ be a small tubular neighbourhood of $k$ in $S^{3}$. Let us call $V_{1}=p^{-1}\left(D^{1}\right) \times S^{1}$, $V_{2}=T \times W_{2} . M_{1}-V_{1}=\left(M_{G}-p^{-1}\left(D_{1}\right)\right) \times S^{1}$ and $\pi_{1}\left(M_{G}-p^{-1}\left(D_{1}\right)\right.$ has the same generators as $\pi_{1}\left(M_{G}\right)$ and the same relations except from the one that assures that the loop $\hat{\alpha}$, a lift of $\alpha=\partial D_{1}$, is vanishing. $\pi_{1}\left(M_{2}-V_{2}\right)$ is the free group generated by $a, b$ and by the loop $\beta=\partial \bar{D}_{2}$ generating the homotopy of $S^{3}-W_{2}$. Now we see that the loop $s$ generating the homotopy of $S^{1}$ in $\left(M_{G}-V_{1}\right) \times S^{1}$ goes to a curve isotopic to $k+l_{3} \beta$. The curves $a, b \subset T \times\{x\} \subset M_{2}-V_{2}$ are seen as the correspondent simple curves generating the homology of a fibre over a point in $\partial D_{1}$ plus some multiple of $\hat{\alpha}$. Finally, the loops $\hat{\alpha}$ and $\beta$ are the same.

We cannot be sure that the result is desired manifold because we cannot conclude that $\hat{\alpha}$ is contractible, but we have turned our initial problem of killing the generator of the homotopy of $S^{1}$ in $M_{G} \times S^{1}$ into a problem that amounts to killing a curve in a manifold whose topology we know quite well.

In $M_{G}$, we consider $T_{2}$, the torus generated by the loops $\hat{\alpha}+a, b . T_{2}$ is a symplectic torus trivially embedded (the symplectic structure on $p^{-1}(0) \times D_{1+\epsilon}^{2}$ can be assumed to be the product symplectic structure). Applying Gompf's construction to $M_{G}$ and a rational elliptic surface along the normal directions of $T_{2}$ and a regular fibre we get a symplectic manifold $\tilde{M}_{G}$. It is clear that $\pi_{1}\left(\tilde{M}_{G}\right)=\pi_{1}\left(M_{G}\right)$, but in $\tilde{M}_{G}$ we have a disk that bounds $\hat{\alpha}$ lying in $\tilde{M}_{G}-p^{-1}\left(D_{1}\right)$. Thus, if we construct the fibre connected sum of $\tilde{M}_{G} \times S^{1}$ and $T \times S^{3}$ along $P_{1}$ and $P_{2}\left(T=p^{-1}(0)\right.$ is of course in $\left.\tilde{M}_{G}\right)$, we get a Poisson manifold $\tilde{M}_{1} \#_{\left(l_{1}, l_{2}, l_{3}\right)} M_{2}$ such that $\pi_{1}\left(\tilde{M}_{1} \#_{\left(l_{1}, l_{2}, l_{3}\right)} M_{2}\right) \cong G$. It is worth noticing that the diffeomorphism type of $\tilde{M}_{1} \#{ }_{\left(l_{1}, l_{2}, l_{3}\right)} M_{2}$ depends at most on $l_{3}$. To see this we observe that $M_{2}-V_{2}$ is a tubular neighbourhood of $T \times \hat{\beta}$, where $\hat{\beta}$ is a loop in the interior of $M_{2}-V_{2}$ isotopic to $\beta$ and thus $\partial\left(M_{2}-V_{2}\right)$ has an $S^{1}$-bundle structure (over $T \times \hat{\beta}$ ) . Hence the diffeomorphism type of the connected sum is totally determined by the image in $\partial\left(\tilde{M}_{1}-V_{1}\right)$ of the $S^{1}$-bundle structure of $\partial\left(M_{2}-V_{2}\right)$ (because $\tilde{M}_{1} \#_{\left(l_{1}, l_{2}, l_{3}\right)} M_{2}$ is the result of collapsing to a point the fibres of the described fibration), and these fibrations are classified by the value of $l_{3}$ (the authors do not know whether different values of $l_{3}$ yield different diffeomorphism types). 
As we have already observed, if we use Kummer surfaces instead of rational elliptic ones to construct $\tilde{M}_{G}$, both $\tilde{M}_{1}$ and $M_{2}$ can be given spin structures and for any such structures, since $H^{2}\left(P_{i} ; \mathbb{Z}\right)$ has no torsion, one can find integers $\bar{l}_{1}, \bar{l}_{2}, \bar{l}_{3}$ with $\tilde{M}_{1} \#\left(\bar{l}_{1}, \bar{l}_{2}, \bar{l}_{3}\right) M_{2}$ admitting a spin structure extending any given ones.

Remark: In the examples above (dimension 5) there are three kinds of symplectic leaves. We have a family parametrized by $S^{1}$ which are diffeomorphic to $\tilde{M}_{G}-T_{1}$ and hence have $G$ as fundamental group; we have another $S^{1}$-family of leaves diffeomorphic to $\mathbb{R}^{2} \times T_{1}$ and both families fill open connected sets separated by a compact leaf $T_{1} \times T$, where $T$ is the closed torus of the Reeb foliation of $S^{3}$. Any of the non-closed leaves has the closed one as set of accumulation points.

Acknowledgments: The authors are grateful for the support of the MCyT programmes PB98/0861, BFM2001-2772. DMT acknowledges the support of an FPI research grant of the Spanish Ministry of Education. The authors would also like to thank Fran Presas for his comments and suggestions, the referee for constructive criticism and Mike Crampin for his help in preparing the final version of this paper. 


\section{References}

[1] M. Bertelson. Foliations associated to regular Poisson structures. Ph. D. Thesis, Stanford University, June 2000.

[2] S. K. Donaldson. Symplectic submanifolds and almost-complex geometry. J. Diff. Geom., 44 , 666-705 (1996).

[3] E. Ghys. L'invariant de Godbillon-Vey. Astérisque, 177-178 (1989).

[4] R. E. Gompf. A new construction of symplectic manifolds. Ann. of Maths., 142, 527-595 (1994).

[5] M. Gromov. Pseudoholomorphic curves on symplectic manifolds. Invent. Math., 82, 307-47 (1985).

[6] M. Gromov. Soft and hard symplectic geometry. In proceedings of the ICM at Berkely 1986, vol. 1, 81-98. Am. Math. Soc. Providence RI (1987).

[7] R.C. Kirby. The topology of 4-manifolds. Lect. Notes Maths., 1384. Springer-Verlag (1991).

[8] D. McDuff. Examples of simply-connected non Kählerian manifolds. J. Diff. Geom, 20, 267-277 (1984).

[9] D. McDuff, D. Salamon. Introduction to symplectic topology. Clarendon Press, Oxford (1995).

[10] I. Vaisman. Lectures on Poisson manifolds. Progress in Mathematics, 118, Birkhäuser (1994).

[11] A. Weinstein. The local structure of Poisson manifolds. J. Diff. Geom., 18, 523-57 (1983).

[12] A. Weinstein. The modular automorphism group of a Poisson manifold. J. Geom. Phys., 23, 379-394 (1997).

Departamento de Matemáticas, Universidad Carlos III de Madrid, Avda. DE LA UNIVERSIDAD 30, 28911 LEGANÉS, EsPAÑA

E-mail address: albertoi@math.uc3m.es, dmtorres@math.uc3m.es 